\title{
Three-dimensional interaction force and tunneling current spectroscopy of point defects on rutile $\mathrm{TiO}_{2}(110)$
}

\author{
Mehmet Z. Baykara, ${ }^{1,2,3, a)}$ Harry Mönig, ${ }^{1,2,4,5, b)}$ Todd C. Schwendemann, ${ }^{1,2,4,6}$ \\ Özhan Ünverdi, ${ }^{1,2,7}$ Eric I. Altman, ${ }^{2,4}$ and Udo D. Schwarz ${ }^{1,2,4}$ \\ ${ }^{1}$ Department of Mechanical Engineering and Materials Science, Yale University, New Haven, \\ Connecticut 06520, USA \\ ${ }^{2}$ Center for Research on Interface Structures and Phenomena (CRISP), Yale University, New Haven, \\ Connecticut 06520, USA \\ ${ }^{3}$ Department of Mechanical Engineering and UNAM-Institute of Materials Science and Nanotechnology, \\ Bilkent University, Ankara 06800, Turkey \\ ${ }^{4}$ Department of Chemical and Environmental Engineering, Yale University, New Haven, Connecticut 06520, \\ USA \\ ${ }^{5}$ Institute of Physics and Center for Nanotechnology (CeNTech), University of Münster, \\ Münster 48149, Germany \\ ${ }^{6}$ Physics Department, Southern Connecticut State University, New Haven, Connecticut 06515, USA \\ ${ }^{7}$ Department of Electrical and Electronics Engineering, Yaşar University, Izmir 35100, Turkey
}

(Received 7 December 2015; accepted 4 February 2016; published online 16 February 2016)

The extent to which point defects affect the local chemical reactivity and electronic properties of an oxide surface was evaluated with picometer resolution in all three spatial dimensions using simultaneous atomic force/scanning tunneling microscopy measurements performed on the (110) face of rutile $\mathrm{TiO}_{2}$. Oxygen atoms were imaged as protrusions in both data channels, corresponding to a rarely observed imaging mode for this prototypical metal oxide surface. Three-dimensional spectroscopy of interaction forces and tunneling currents was performed on individual surface and subsurface defects as a function of tip-sample distance. An interstitial defect assigned to a subsurface hydrogen atom is found to have a distinct effect on the local density of electronic states on the surface, but no detectable influence on the tip-sample interaction force. Meanwhile, spectroscopic data acquired on an oxygen vacancy highlight the role of the probe tip in chemical reactivity measurements. (C) 2016 AIP Publishing LLC. [http://dx.doi.org/10.1063/1.4942100]

In heterogenous catalysis, the atomic-scale specifics of the potential energy landscape experienced by individual molecules interacting with the catalyst surface are of fundamental and technological importance. ${ }^{1}$ Particularly interesting in this context is how point defects such as vacancies and interstitials influence the local chemical reactivity. ${ }^{2}$ As such, probing the structural and electronic properties of catalyst surfaces on the atomic scale constitutes an important step towards understanding and controlling catalytic activity. The method of three-dimensional atomic force microscopy (3D-AFM) allows the recording of volumetric maps of the tip-sample interaction force with picometer-scale spatial and piconewton-scale force resolution. ${ }^{3,4}$ The combination of the method with the complementary recording of tunneling current (3D-AFM/STM) leads to the ability to simultaneously probe chemical interactions as well as electronic properties with local specificity as a function of tip-sample distance, making it ideal for fundamental catalysis research. ${ }^{5,6}$

Transition metal oxides are widely used as catalysts. ${ }^{7,8}$ Among the large family of transition metal oxide surfaces, the (110) face of rutile titanium dioxide $\left(\mathrm{TiO}_{2}\right)$ is considered prototypical due to the fact that the types of defects commonly encountered on this surface are well known. ${ }^{9}$ Combined realspace measurements of atomic-scale structure, site-specific

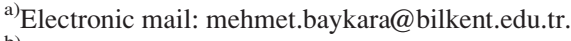

b)Electronic mail: harry.moenig@uni-muenster.de.
}

chemical interactions, and local electronic properties on this surface are, however, still lacking.

As shown in Fig. 1(a), the rutile (110) surface consists of alternating rows of $\mathrm{Ti}^{4+}$, in-plane $\mathrm{O}^{2-}$, as well as bridging $\mathrm{O}^{2-}$ ions. Regular defects on the $\mathrm{TiO}_{2}(110)$ surface include bridging $\mathrm{O}^{2-}$ vacancies and $\mathrm{H}$ atoms adsorbed on bridging $\mathrm{O}^{2-}$ (referred to as $\mathrm{OH}$ groups in the following). Another type of defect that has been identified via combined AFM/STM imaging is an interstitial in the form of a subsurface $\mathrm{H}$ atom, as shown in Fig. 1(b). ${ }^{10}$ Despite the aforementioned previous characterization work, the specific roles that these defects play in surface chemistry are still under debate. One way to address this issue is through direct quantification of their chemical reactivity via site-specific force spectroscopy experiments; results from such experiments are, however, still rare. ${ }^{11}$ This letter presents 3D-AFM/STM measurements aimed at answering these questions. In contrast to prior force spectroscopy studies on $\mathrm{TiO}_{2}(110)$ based on individual force curves,${ }^{11}$ 3D-AFM/STM maps allow the direct study of how individual defects affect interaction forces, the work function, and local density of electronic states (LDOS), inferred from spatial changes in tunneling current measurements; as a function of both lateral and vertical distance from the defect's location.

To obtain a clean $\mathrm{TiO}_{2}(110)$ surface, the sample was prepared via repeated sputter/anneal cycles in ultrahigh vacuum with a base pressure below $5 \times 10^{-10} \mathrm{mbar}$. The $3 \mathrm{D}$ AFM/STM experiments were performed with a home-built 



(b) [110]

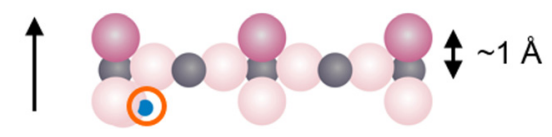

FIG. 1. (a) Structural model illustrating the rutile $\mathrm{TiO}_{2}(110)$ surface as well as the two most commonly observed defects: Oxygen vacancies (red circle) and $\mathrm{OH}$ groups (green circle). (b) Side view of the $\mathrm{TiO}_{2}(110)$ surface highlighting a subsurface $\mathrm{H}$ atom (orange circle). The depicted arrangement of this defect follows predictions from density functional theory simulations. ${ }^{10}$

system $^{12}$ at a temperature of $T=77 \mathrm{~K}$. A quartz tuning fork with a field ion beam-treated $\mathrm{Pt} / \mathrm{Ir}$ tip was used as the force/ tunneling current sensor (spring constant $k \approx 2000 \mathrm{~N} / \mathrm{m}$, resonance frequency $f_{0}=25328 \mathrm{~Hz}$ ). For the $3 \mathrm{D}-\mathrm{AFM} / \mathrm{STM}$ data set presented in this letter, 27 images were acquired with active frequency shift $(\Delta f)$ feedback at evenly spaced $\Delta f$ set points between $-1.45 \mathrm{~Hz}$ and $-1.10 \mathrm{~Hz}$ (oscillation amplitude $A \approx 1.50 \mathrm{~nm}$, bias voltage $U=+1.6 \mathrm{~V}$ applied to the sample). The three-dimensional $\Delta f(x, y, \mathrm{z})$ data have been converted to force using previously discussed data processing procedures. ${ }^{13,14}$ In particular, note that the use of the 3DAFM/STM approach prevents feedback-induced cross-talk between force and tunneling current channels, ${ }^{15}$ which is a considerable advantage compared to previous studies performed on the $\mathrm{TiO}_{2}(110)$ surface via the recording of individual AFM/STM maps under active feedback. ${ }^{16}$

Before the specifics of the 3D-AFM/STM data are discussed, the basic contrast modes observed on the $\mathrm{TiO}_{2}(110)$ surface are reviewed. While the overwhelming majority of STM images reported in the literature feature bright stripes corresponding to rows of $\mathrm{Ti}^{4+},{ }^{17}$ three imaging modes are commonly encountered during AFM experiments: ${ }^{18}$ (1) the protrusion mode, where $\mathrm{Ti}^{4+}$ rows are imaged as bright stripes and regular defects as bright spots between them; (2) the hole mode, where bridging $\mathrm{O}^{2-}$ rows are imaged as bright stripes and regular defects as dark spots on top; and (3) the neutral mode, where bridging $\mathrm{O}^{2-}$ rows are imaged as bright stripes, $\mathrm{O}^{2-}$ vacancies are imaged as dark depressions, and $\mathrm{OH}$ groups as bright protrusions. In combined AFM/STM measurements, all three modes can be observed in the AFM channel, with either in-phase or out-of-phase imaging of bright rows in the STM channel. ${ }^{16}$ While a straightforward assignment of electrostatic polarity to tip apices (negative, positive, and neutral, respectively) seemed initially to explain the three contrast modes, ${ }^{18,19}$ detailed force spectroscopy experiments with support from $a b$ initio calculations eventually allowed assignment of the observed contrasts to realistic tip apex structures, which are typically terminated by clusters of $\mathrm{Ti}$ and $\mathrm{O}$ atoms picked up from the surface. ${ }^{11}$

Figure 2 shows the constant-height force and tunneling current data at the plane of closest approach in the 3D-AFM/ STM data set. Based on the depression-type defect in the upper right corner of the image, the contrast in the force channel (Fig. 2(a)) can be explained by either "hole" or "neutral" mode imaging, since both modes exhibit bright rows assigned to bridging $\mathrm{O}^{2-}$ sites. In hole mode imaging, the defect on the upper right corner could either be an $\mathrm{O}^{2-}$ vacancy or an $\mathrm{OH}$ group. On the other hand, in neutral mode imaging, which is expected to closely resemble the atomic-scale topography of the sample surface, this defect would have to be an $\mathrm{O}^{2-}$ vacancy. ${ }^{18,20}$ To distinguish which imaging mode and consequently what sort of tip apex is responsible for the experimental data, we investigated the topographical corrugation of the $\mathrm{O}^{2-}$ rows for the highest interaction force in our $3 \mathrm{D}$ data set $(0.77 \mathrm{nN})$. With the mean corrugation of $\mathrm{O}^{2-}$ rows $(\sim 0.4 \AA)$ significantly less than the actual height of bridging $\mathrm{O}^{2-}$ sites above in-plane $\mathrm{Ti}^{4+}(\sim 1 \AA),{ }^{9}$ the possibility of a positively-terminated, reactive tip apex leading to hole-mode imaging can be excluded; consequently, the imaging mode must be of the neutral variety, and the defect on the upper right corner is identified as an $\mathrm{O}^{2-}$ vacancy. It should be indicated that the value of $0.4 \AA$ observed in our experiments for the corrugation of $\mathrm{O}^{2-}$ rows is in remarkable agreement with the values reported in the literature for a neutral tip ${ }^{18,20}$ and smaller than the values reported for positively-terminated tips $(\geq 0.5$ $\AA$ ). ${ }^{18}$ Finally, while the actual structure of the tip apex cannot be determined in a straightforward fashion, a comparison with results in the literature points to an $\mathrm{OH}$-terminated apex as a likely candidate. ${ }^{20}$

Comparing the constant-height force map of Fig. 2(a) with the simultaneously recorded tunneling current data (Fig. 2(b)), we see that the tunneling current maxima coincide with the bright rows of the force map - and thus the $\mathrm{O}^{2-}$ sites - in this particular experiment. Even though the imaging of $\mathrm{O}^{2-}$ rows as bright in STM images has been predicted for small tip-sample distances earlier, ${ }^{21}$ we note that this is a rarely found contrast mode (described thus far only once for combined AFM/STM experiments carried out with what was presumably a neutral $\operatorname{tip}^{16}$ ), as the LDOS in the conduction band probed at positive sample biases are mostly concentrated at $\mathrm{Ti}^{4+}$ surface sites. ${ }^{9}$

Having discussed the contrast in both force and tunneling current channels, the peculiar feature visible within the dashed orange circle in the lower-left part is considered next. This defect manifests as a bright "double protrusion" on an $\mathrm{O}^{2-}$ row in the current channel that "bleeds" into an adjacent $\mathrm{O}^{2-}$ row without an apparent signature in force; the threedimensional representation in the inset of Fig. 2(b) more clearly highlights the characteristic double protrusion and bleeding into the neighboring row. A similar structural element has been reported before, where atomic-scale protrusions were 

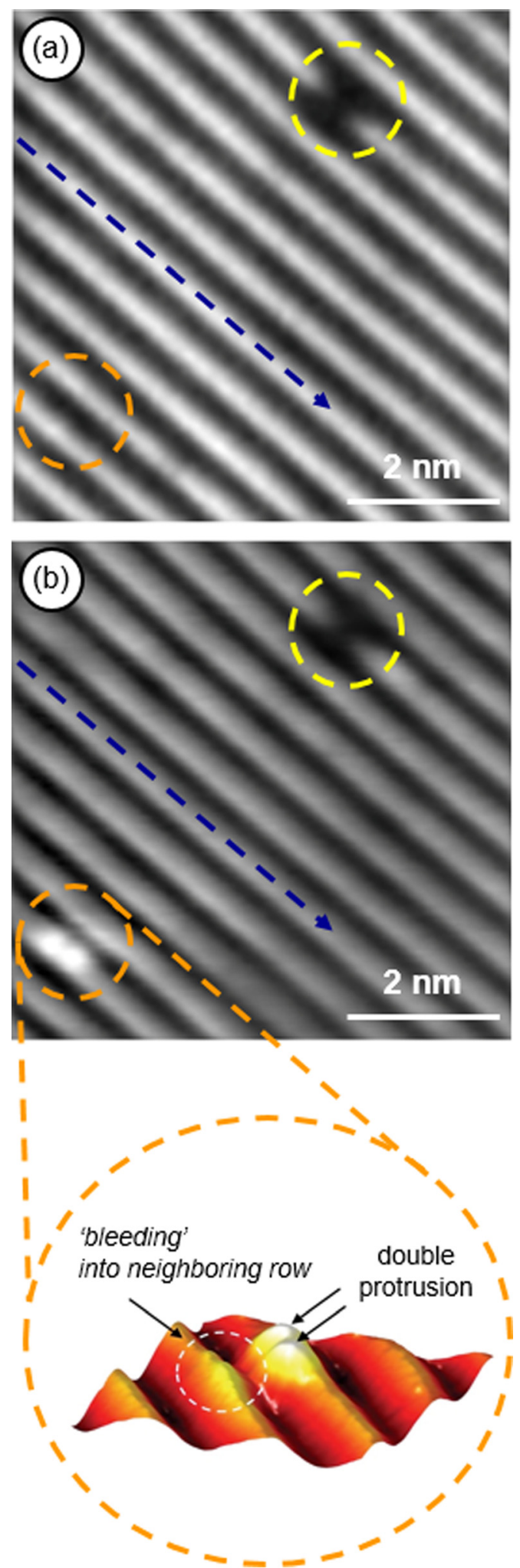

FIG. 2. Simultaneously recorded force (a) and tunneling current (b) images extracted from the 3D-AFM/STM data set at the plane of closest approach (average attractive force: $816 \mathrm{pN}$; force contrast: $100 \mathrm{pN}$; average current: $183 \mathrm{pA}$; current contrast: $340 \mathrm{pA}$ ). The positions of the $\mathrm{O}^{2-}$ vacancy and the subsurface $\mathrm{H}$ atom are highlighted by the dashed yellow and orange circles, respectively. As indicated by the dashed blue line, the tunneling current image is in-phase with the force contrast, meaning that bridging $\mathrm{O}^{2-}$ sites are maxima for both interaction force and tunneling current. The inset features a three-dimensional representation of the tunneling current around the subsurface defect (average current: $199 \mathrm{pA}$, current contrast: $368 \mathrm{pA}$, image size: $1.4 \mathrm{~nm} \times 1.4 \mathrm{~nm}$ ); note that the viewing direction is rotated compared to (b) to allow a better view onto the "bleeding" of the defect into the neighboring $\mathrm{O}^{2-}$ row (highlighted by the dashed white circle).
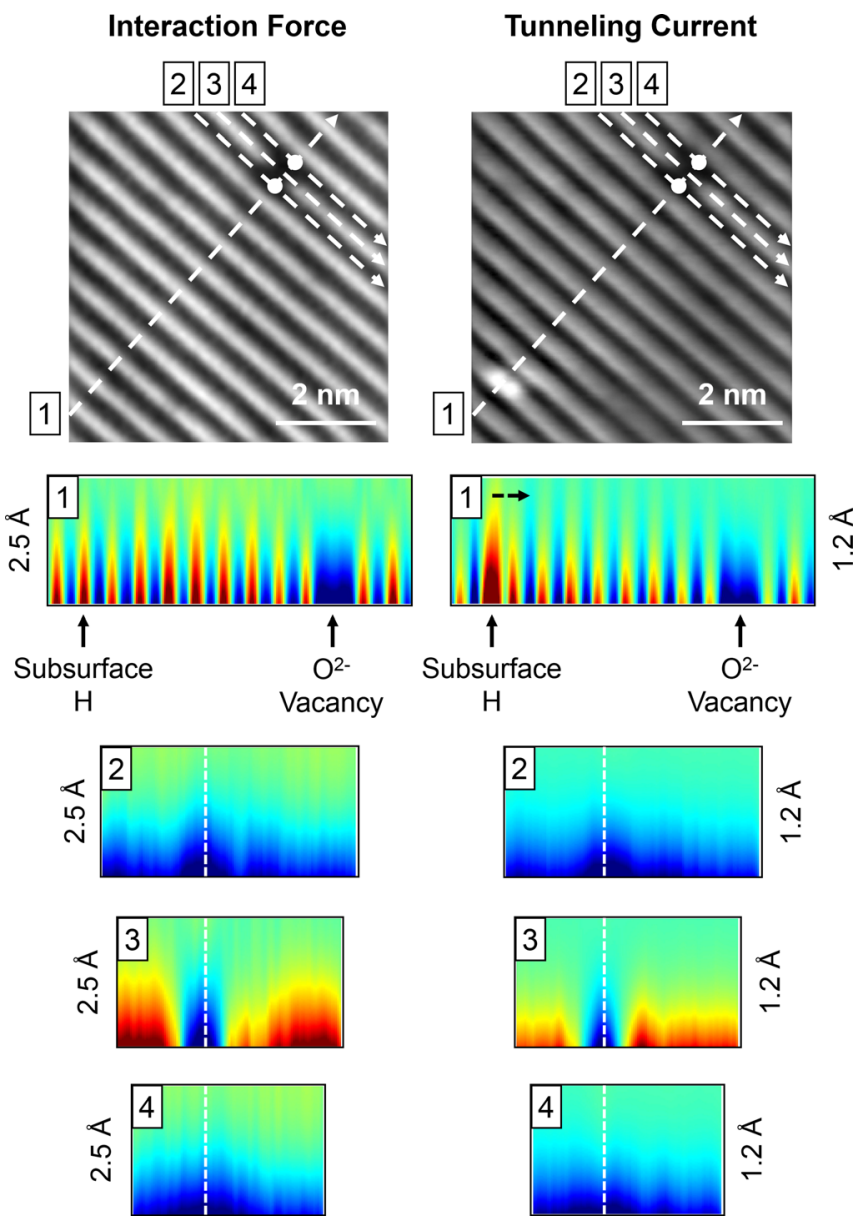

FIG. 3. Two-dimensional interaction force and tunneling current slices extracted from the 3D-AFM/STM data set along directions indicated in the top panel by 1 to 4 . The color scale for force slices is between $+30 \mathrm{pN}$ (dark $\mathrm{red} /$ more attractive) and $-30 \mathrm{pN}$ (dark blue/less attractive); note that absolute force values are plotted and that the average absolute force at each height, which decreases from $816 \mathrm{pN}$ to $604 \mathrm{pN}$, has been subtracted to ensure best visibility of the force contrast. ${ }^{3}$ For current slices, the color scale ranges from $+100 \mathrm{pA}$ (dark red) to $-80 \mathrm{pA}$ (dark blue); similarly as for the force, the average current, which decreases from $183 \mathrm{pA}$ to $15 \mathrm{pA}$, has been subtracted at each height. The dashed black arrow highlights the asymmetric influence of the defect on the local density of states. The $\mathrm{O}^{2-}$ vacancy has a clearly noticeable influence on interaction force and tunneling current on neighboring rows of $\mathrm{Ti}^{4+}$ (see the interaction force and tunneling current slices denoted by 2 and 4, and the dashed white line indicating the positions corresponding to the $\mathrm{O}^{2-}$ vacancy in both channels, which are further highlighted by white dots in the force and tunneling current images).

observed on top of $\mathrm{O}^{2-}$ rows in the STM channel without an observable trace in AFM maps. ${ }^{10}$ By relying on a combination of time-lapse AFM/STM imaging and density functional theory (DFT) simulations, these features have been assigned to subsurface interstitial $\mathrm{H}$ atoms. In addition, such subsurface $\mathrm{H}$ defects are expected to lead to an asymmetric perturbation of LDOS towards one of the Ti rows next to them, which is clearly observed in our tunneling current image as well (referred to as "bleeding" above). As a consequence, we assign this feature to a subsurface $\mathrm{H}$ atom. It should be noted that DFT calculations also predict the observed appearance of the defect as a double protrusion on one of the neighboring $\mathrm{Ti}$ rows, which could not be resolved in earlier work. ${ }^{10}$ In contrast, the improved spatial resolution provided by the lowtemperature instrument used in this study and the increased sensitivity to short-range interactions due to low-amplitude 


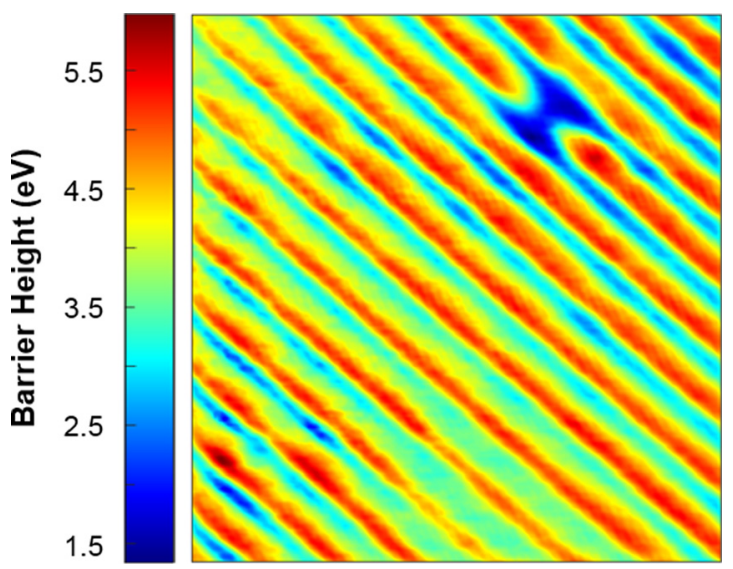

FIG. 4. Tunneling barrier height map corresponding to the region of the $\mathrm{TiO}_{2}$ surface covered in the 3D-AFM/STM data set.

operation allow us to clearly identify a "double protrusion" feature, albeit on the $\mathrm{O}^{2-}$ row over the subsurface defect.

In addition to providing quantitative information on the electronic and chemical signatures of certain structural features at a constant distance, one of the particular strengths of 3D-AFM/STM data sets is that they also allow the study of interaction force and tunneling current as a function of the vertical position on any given surface spot. Figure 3 describes such an analysis, where 2D cross-sections of force and tunneling current acquired along certain directions (denoted by 1-4) in the vicinity of the point defects are presented. It can be seen that while the force contrast does not disappear until a height of about $150 \mathrm{pm}$ from the plane of closest approach, lateral fluctuations in the tunneling current fade out at about half that distance. On the other hand, tunneling currents detected over the subsurface defect site extend about $40 \mathrm{pm}$ further out of the surface when compared to the unperturbed regions of the sample. The reason behind this latter observation could be two-fold: (a) an increase in LDOS and/or (b) a local reduction in work function and, thus, the barrier height for tunneling. Fortunately, the 3D-AFM/STM data set presented here contains sitespecific information on the dependence of tunneling currents on tip-sample separation, from which a barrier height map can be readily constructed (Fig. 4). No reduction in barrier height is detected in the vicinity of the subsurface defect; if anything, a slight increase is noted that is not significant compared to the standard deviation of barrier height values observed on $\mathrm{O}^{2-}$ rows. Therefore, it can be concluded that an increase in LDOS is responsible for the prolonged detection of tunneling currents with increasing tip-sample distance over the subsurface defect.

Remarkably, the 2D cross-sections of interaction force and tunneling current recorded over Ti rows next to the $\mathrm{O}^{2-}$ row with the vacancy (sections 2 and 4 in Fig. 3) reveal that the effect of the defect extends over lateral distances of at least $3.25 \AA$ (the spacing between adjacent $\mathrm{O}$ and Ti rows on the $\mathrm{TiO}_{2}(110)$ surface). Additionally, the distinctly asymmetric effect of the subsurface $\mathrm{H}$ atom on the LDOS distribution in its vicinity is visualized by the corresponding tunneling current slice 1 . While the force channel does not exhibit any perturbations around the subsurface $\mathrm{H}$ defect at any height, the interaction appears significantly less attractive above the $\mathrm{O}^{2-}$ vacancy in the top right corner of the image. While this result may seem confusing at first since defects on metal oxide surfaces are generally thought to be active sites expected to exhibit high chemical interaction forces ${ }^{22}$ the underlying reason becomes clear when (i) the neutral character of the tip apex is considered and (ii) it is taken into account that images reflect forces at constant height above the surface plane, leading to an increased tip-sample distance (by $\sim 1 \AA$, see Fig. 1(b)) over the vacancy when compared to the rest of the $\mathrm{O}^{2-}$ row. Our results underline that the chemical reactivity of specific atomic sites on a catalytic surface should always be considered with respect to the atomic/molecular species that it is expected to interact with, thereby suggesting that future expansions of our approach would ideally include molecule-specific investigations of surface chemical reactivity via the utilization of functionalized AFM probes. ${ }^{23}$

In summary, we have presented three-dimensional, atomic-resolution measurements of interaction force and tunneling current on the prototypical metal oxide surface of rutile $\mathrm{TiO}_{2}(110)$. In particular, point defects including an $\mathrm{O}^{2-}$ vacancy and a subsurface $\mathrm{H}$ atom have been evaluated in terms of their effect on local interaction forces and electronic density of states as a function of lateral and vertical position on the sample surface. Simultaneous measurements of interaction forces and tunneling currents over the subsurface $\mathrm{H}$ atom have underlined the fact that interaction forces measured via AFM are not necessarily coupled to the surface's LDOS, but may often provide physically relevant information that is complementary to the information contained in tunneling current data. ${ }^{5}$ Remarkably, the high spatial resolution of our data set allowed us to confirm theoretical predictions regarding the effect of the subsurface $\mathrm{H}$ atom on the LDOS as detected by tunneling current measurements. ${ }^{10}$ Our results demonstrate the applicability of the 3D-AFM/STM method to the detailed investigation of structural, chemical, and electronic properties of catalytically important material surfaces in real space with high resolution.

Financial support from the National Science Foundation through the Yale Materials Research Science and Engineering Center (Grant No. MRSEC DMR-1119826) and the Materials World Network program (Grant No. MWN DMR-0806893) and the U.S. Department of Energy (Basic Energy Sciences Grant No. DE-FG02-06ER15834) are gratefully acknowledged. H.M. acknowledges support by the Deutsche Forschungsgemeinschaft through the transregional collaborative research center TRR 061 (Project B7). M.Z.B. acknowledges support by the Outstanding Young Scientist program of the Turkish Academy of Sciences (TÜBA-GEBIP).

${ }^{1} \mathrm{~J}$. M. Thomas and W. J. Thomas, Principles and Practice of Heterogenous Catalysis (Wiley-VCH, Weinheim, 2015).

${ }^{2}$ H. J. Freund, Chem. Eur. J. 16, 9384 (2010).

${ }^{3}$ B. J. Albers, T. C. Schwendemann, M. Z. Baykara, N. Pilet, M. Liebmann, E. I. Altman, and U. D. Schwarz, Nat. Nanotechnol. 4, 307 (2009).

${ }^{4}$ M. Z. Baykara, T. C. Schwendemann, E. I. Altman, and U. D. Schwarz, Adv. Mater. 22, 2838 (2010).

${ }^{5}$ M. Z. Baykara, M. Todorović, H. Mönig, T. C. Schwendemann, Ö. Ünverdi, L. Rodrigo, E. I. Altman, R. Pérez, and U. D. Schwarz, Phys. Rev. B 87, 155414 (2013). 
${ }^{6}$ E. I. Altman, M. Z. Baykara, and U. D. Schwarz, Acc. Chem. Res. 48, 2640 (2015).

${ }^{7}$ V. E. Henrich and P. A. Cox, The Surface Science of Metal Oxides (Cambridge University Press, Cambridge, 1994).

${ }^{8}$ M. A. Henderson, Surf. Sci. Rep. 66, 185 (2011).

${ }^{9}$ U. Diebold, Surf. Sci. Rep. 48, 53 (2003).

${ }^{10}$ G. H. Enevoldsen, H. P. Pinto, A. S. Foster, M. C. R. Jensen, W. A. Hofer, B. Hammer, J. V. Lauritsen, and F. Besenbacher, Phys. Rev. Lett. 102, 136103 (2009).

${ }^{11}$ A. Yurtsever, D. Fernández-Torre, C. González, P. Jelínek, P. Pou, Y. Sugimoto, M. Abe, R. Pérez, and S. Morita, Phys. Rev. B 85, 125416 (2012).

${ }^{12}$ B. J. Albers, M. Liebmann, T. C. Schwendemann, M. Z. Baykara, M. Heyde, M. Salmeron, E. I. Altman, and U. D. Schwarz, Rev. Sci. Instrum. 79, 033704 (2008).

${ }^{13}$ B. J. Albers, T. C. Schwendemann, M. Z. Baykara, N. Pilet, M. Liebmann, E. I. Altman, and U. D. Schwarz, Nanotechnology 20, 264002 (2009).

${ }^{14}$ M. Z. Baykara, O. E. Dagdeviren, T. C. Schwendemann, H. Mönig, E. I. Altman, and U. D. Schwarz, Beilstein J. Nanotechnol. 3, 637 (2012).
${ }^{15}$ M. Z. Baykara, M. Todorović, H. Mönig, T. C. Schwendemann, L. Rodrigo, E. I. Altman, R. Pérez, and U. D. Schwarz, J. Phys. Chem. C 119, 6670 (2015).

${ }^{16}$ G. H. Enevoldsen, H. P. Pinto, A. S. Foster, M. C. R. Jensen, A. Kuhnle, M. Reichling, W. A. Hofer, J. V. Lauritsen, and F. Besenbacher, Phys. Rev. B 78, 045416 (2008).

${ }^{17}$ U. Diebold, J. F. Anderson, K. O. Ng, and D. Vanderbilt, Phys. Rev. Lett. 77, 1322 (1996).

${ }^{18}$ G. H. Enevoldsen, A. S. Foster, M. C. Christensen, J. V. Lauritsen, and F. Besenbacher, Phys. Rev. B 76, 205415 (2007).

${ }^{19}$ J. V. Laurtisen, A. S. Foster, G. H. Olesen, M. C. Christensen, A. Kühnle, S. Helveg, J. R. Rostrup-Nielsen, B. S. Clausen, M. Reichling, and F. Besenbacher, Nanotechnology 17, 3436 (2006).

${ }^{20}$ R. Bechstein, C. Gonzalez, J. Schütte, P. Jelinek, R. Perez, and A. Kühnle, Nanotechnology 20, 505703 (2009).

${ }^{21}$ R. E. Tanner, M. R. Castell, and G. A. D. Briggs, Surf. Sci. 412/13, 672 (1998).

${ }^{22}$ T. König, G. H. Simon, U. Martinez, L. Giordano, G. Pacchioni, M. Heyde, and H. J. Freund, ACS Nano 4, 2510 (2010).

${ }^{23}$ L. Gross, F. Mohn, N. Moll, P. Liljeroth, and G. Meyer, Science 325, 1110 (2009). 